\title{
LIGO optical coatings pose new challenges in materials research
}

\section{Rachel Berkowitz}

$\mathrm{W}$ hen the Laser Interferometer Gravitational-Wave Observatory (LIGO) announced the first ever detection of a gravitational wave in September 2015, one common question was "when will they find the next one?"

The answer came quickly, with a second detection on December 25, causing excitement throughout the multinational

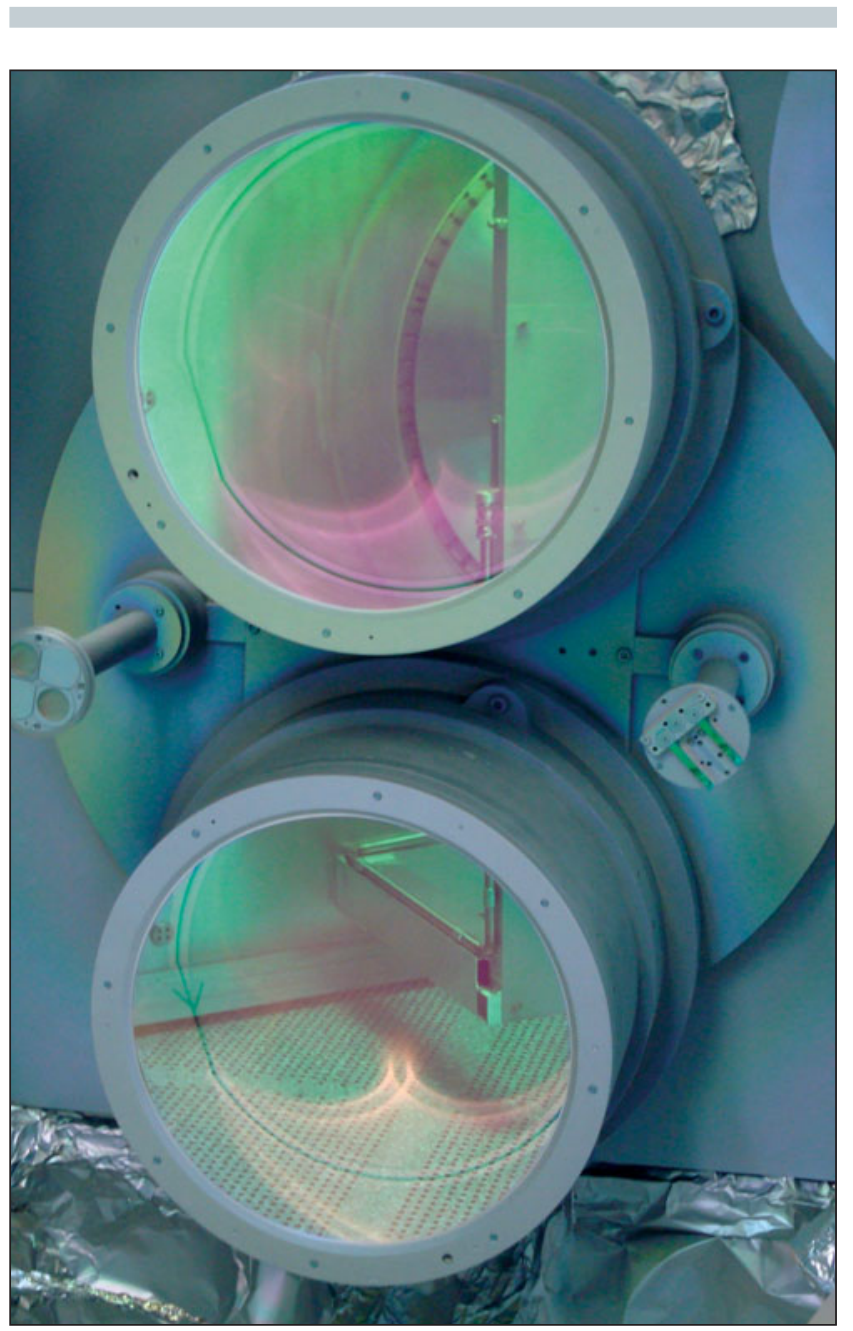

Two LIGO test masses after coating in the ion-beam sputtering deposition chamber at the Laboratoire des Matériaux Avancés (LMA) in Lyon, France. Credit: LMA. collaboration that designed and built the intricate optical instruments.

Gravitational waves, or "ripples in spacetime," radiate throughout the universe for billions of light-years, and were predicted a century ago by Einstein's general theory of relativity. They are a byproduct of vast amounts of energy generated by massive, accelerating bodies such as supernovae or merging black holes.

Not only has the discovery opened a new era of astronomy, it has also unveiled new horizons for optical and materials sciences.

In the search for gravitational waves, LIGO's optics system plays a critical role. The interferometer amplifies a laser beam and splits it into two beams that are sent down 4-km-long orthogonal vacuum tubes, or arms. The beams build power by resonating between some of the world's highest-quality mirrors, or "test masses," suspended at either end of each arm. Specially designed coatings on the surfaces of the fused-silica test masses make them highly reflective, thus controlling the laser beam's path within the interferometers.
When the split beams recombine, they produce a signal that is observable at the photodetector. Any minute change in one arm's path length produces a shift in the detected light power. Thus a difference in arm length can be measured with unprecedented precision - the system is capable of measuring a relative length shift less than one thousandth the diameter of a proton. The passing of a gravitational wave, as detected simultaneously by two facilities in Livingston, La., and Hanford, Wash., creates a spatial shift on the order of just $10^{-18}$ meters.

However, vibrations and environmental noise reduce sensitivity of the entire system. Actual strain on the instruments at all frequencies must be distinguished from gravitational-wave signals, and the dielectric coatings which give the test masses their reflectivity and low optical loss contribute to this noise.

"A better understanding of coatings that will not jeopardize optical properties has great potential to improve LIGO sensitivity," says Fred Raab, Director of the LIGO Hanford Observatory.

At frequencies below $15 \mathrm{~Hz}$, LIGO's sensitivity is limited by seismic motion. Above $200 \mathrm{~Hz}$, "shot" noise, or the quantum fluctuations in the detected laser power, is the limiting factor. But the mid-frequency range from $\sim 30$ to $200 \mathrm{~Hz}$, where many gravitational-wave signals propagate and other noise sources are minimal, is limited by thermal noise from the coatings. Thermal noise refers to the materials' inherent molecular motion, manifested as mechanical loss, which causes vastly more movement in the interferometers than that produced by a gravitational wave.

"We understand and can correct for the high- and low-frequency, and some intermediate noise sources. But we're stuck with thermal," Raab says. 


\section{Low-loss materials}

When the LIGO Scientific Collaboration first designed the instruments, one goal was to identify a mirror coating that minimized optical loss, thus ensuring that all laser light resonates within the interferometer arms. A poor choice of coating could allow light to escape, or be absorbed by and heat the mirrors. Of the available low-absorption materials that would meet the required sensitivity, optical coating vendors suggested tantalum pentoxide $\left(\mathrm{Ta}_{2} \mathrm{O}_{5}\right)$ and silica $\left(\mathrm{SiO}_{2}\right)$ layers.

Alternating layers of high- and low-refractive-index materials optimize the coating structure for reflectivity and absorption. Silica has a lower index and lower mechanical loss, while tantala has a higher index and higher mechanical loss. Both have relatively low absorption. While thermal noise is best reduced by reducing tantala thickness, doing so requires more layers to achieve the necessary reflectivity.

"The idea of optimizing is the observation that the tantala is most problematic for thermal noise," says Gregg Harry, a physicist now at American University in Washington, DC, and a member of the LIGO Scientific Collaboration.

Key to these materials' suitability is their amorphous, or noncrystalline, structure. Techniques exist to deposit them in smooth layers over large surface areas.

\section{Perfect uniformity}

The first version of the instruments, or "Initial LIGO," operated from 2001 to 2010. Then, they underwent major hardware and software upgrades to a more sensitive "Advanced LIGO" design, which surpassed "Initial LIGO" sensitivity in 2015. Advanced LIGO made headlines by detecting the first gravitational wave shortly after it began operations in 2015. While Advanced LIGO's current sensitivity is 3-4 times greater than Initial LIGO, modifications continue toward reaching its maximum sensitivity, 10 times greater than Initial LIGO.

Research Electro-Optics (REO) in Boulder, Col., deposited the coatings on Initial LIGO's perfectly polished mirrors in the late 1990s. The company had been an early adopter of ion-beam sputtering in the late 1980s, a then-novel and highly
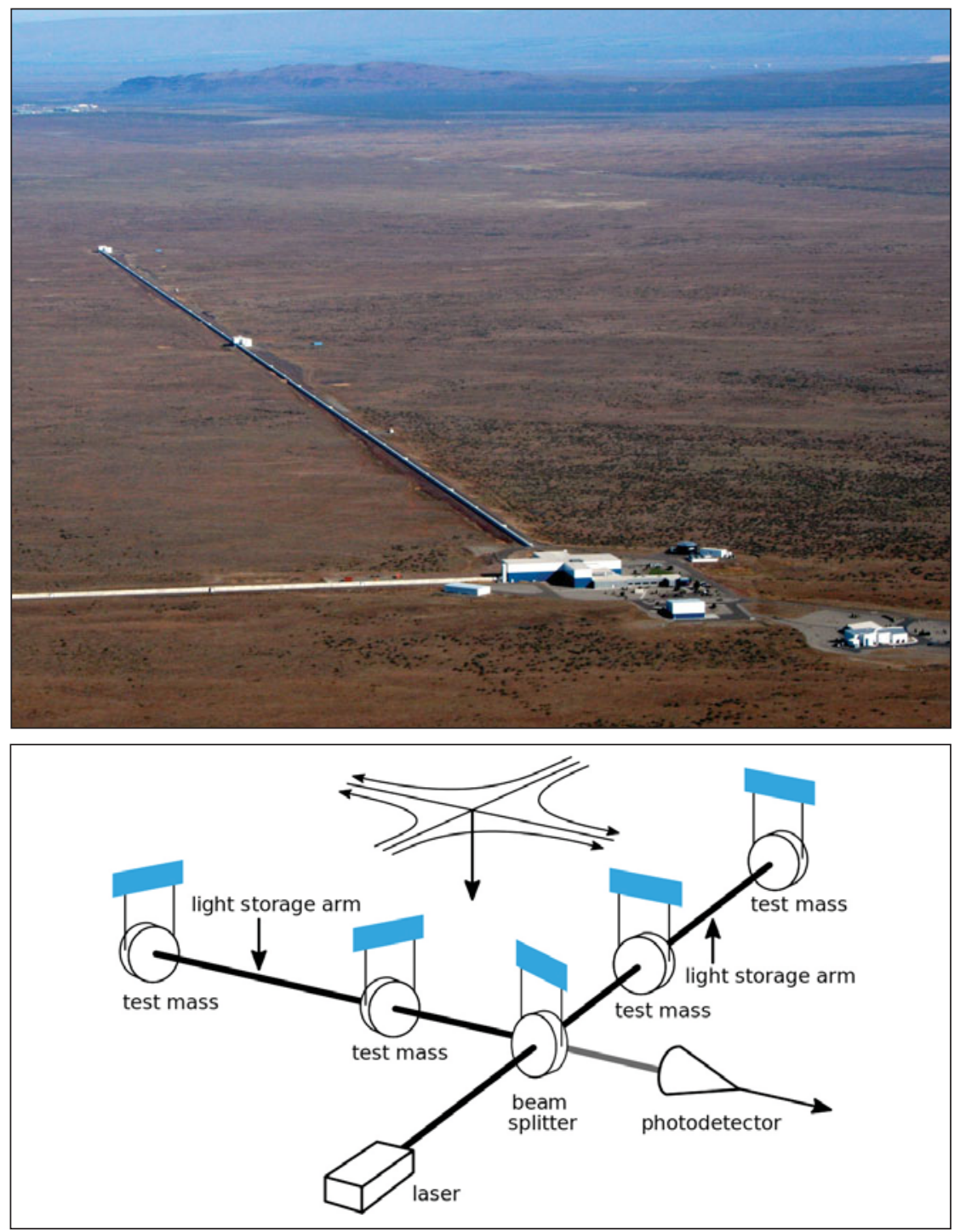

Top: Aerial view of LIGO Hanford Observatory. Credit: LIGO Laboratory. Bottom: Schematic of a laser interferometer. Credit: LIGO Laboratory.

conformal optical coating technique. This allowed REO to build its reputation on producing what were then the world's best low-loss mirrors.

Dale Ness, who served as Project Manager for Initial LIGO optics at REO, developed the mathematical model that proved the feasibility of achieving a highly uniform coating over 25-cm-diameter mirrors. Demonstrating the capability on a smaller sample secured REO the contract to coat the test masses.

Using software developed by H. Angus MacLeod, now Professor Emeritus of Optical Sciences at The University of Arizona, the REO team designed a stack of layers, each tens to a few hundred nanometers thick, to produce the various thinfilm coatings in compliance with stringent requirements of 1-ppm absorption.

Ion-beam sputtering sprays each layer onto the surface in a nonuniform, Gaussian-like plume. Within the spray, the optics are revolved and orbited around each other, like moons around a planet. Achieving high uniformity requires a mask designed specially for the shape of the plume.

"You wind up with a leaf-shaped mask that selectively blocks the material, so you end with uniform thickness all the way across," Ness explains. 
In addition to meeting technical specifications, the team faced the formidable task of handling optics worth a quarter of a million dollars each. "You can imagine horror stories of loading a part and applying the wrong coating, or putting it on the wrong side; human errors happen," Ness says.

But despite these fears, the coated optics emerged error-free, which Ness attributes to egalitarian teamwork and immaculate clean room protocol.

\section{Advanced design}

The Initial LIGO design demonstrated that absorption in the coatings is an important limitation, because absorbed light heats the optics and changes the radius of curvature of the mirror. Laboratory work showed that coating thermal noise would be an important limitation for future detectors. When considering the higher sensitivity requirements for the upgrade to Advanced LIGO, one focus was on reducing absorption in the mirrors.

"The main difference concerns the optical absorption, going from $1 \mathrm{ppm}$ to 0.5 ppm [absorption loss]," says Laurent Pinard, research scientist at Laboratoire des Matériaux Avancés (LMA) in Lyon, France. Scattering loss also had to be reduced to under $10 \mathrm{ppm}$.

LMA's history of research on lowabsorption coatings helped them win the project to design and apply coatings to Advanced LIGO's test masses. JeanMarie Mackowski, then-head of LMA, proposed adding titanium dioxide $\left(\mathrm{TiO}_{2}\right)$ to the tantala layers to increase their refractive index. The atomic size of titanium allows for dense packing within the tantalum and oxygen matrix, while the $\mathrm{TiO}_{2} / \mathrm{Ta}_{2} \mathrm{O}_{5}$ alloy's high melting point confirms its stable amorphous structure.

By doping with titania and annealing, tantala's mechanical loss can be reduced to within a factor of five of silica. Furthermore, Advanced LIGO coatings feature a dichroic design, meaning that they provide some reflectance at a secondary wavelength $(532 \mathrm{~nm})$ used for auxiliary measurements, in addition to the main beam (1064 nm).

"Since the mechanical loss of tantala is much greater than [that of] silica, we use an optimized layer structure rather than the usual quarter wave stack," says Rana Adhikari, physics professor at the California Institute of Technology (Caltech). The usual reflective coating design comprises alternating layers of high- and low-index material, each having a thickness one-quarter wavelength of the incident light. The optimized coatings were introduced and developed in a collaboration among the University of Sannio (Italy), Caltech, and LMA, along with research at the Massachusetts Institute of Technology, the University of Glasgow, and many more.

\section{FOR FURTHER READING}

- G.M. Harry, H. Armandula, E. Black, D.R.M. Crooks, G. Cagnoli, J. Hough, P. Murray, S. Reid, S. Rowan, P. Sneddon, M.M. Fejer, R. Route, S.D. Penn, "Thermal Noise from Optical Coatings in Gravitational Wave Detectors," Appl. Opt. 45, 1569 (2006), doi:10.1364/AO.45.001569.

-D.C. Ness, "Techniques and Strategies for Manufacturing lon Beam Sputtered Coatings for the LIGO I Core Optical Components," in Optical Interference Coatings 2016, OSA Technical Digest Series (online) (Optical Society of America, 2016), paper MB.11, doi:10.1364/OIC.2016.MB.11.

- G.M. Harry, M.R. Abernathy, A.E. Becerra-Toledo, H. Armandula, E. Black, K. Dooley, M. Eichenfield, C. Nwabugwu, A. Villar, D.R.M. Crooks, et al., "Titania-Doped Tantala/Silica Coatings for GravitationalWave Detection," Class. Quantum Grav. 24, 405 (2007), doi:10.1088/0264-9381/24/2/008.

- M. Principe, "Reflective Coating Optimization for Interferometric Detectors of Gravitational Waves," Opt. Express 23, 10938 (2015), doi:10.1364/OE.23.010938.

- R. Bassiri, M.R. Abernathy, F. Liou, M.M. Fejer, "Order, Disorder and Mixing: The Atomic Structure of Amorphous Mixtures of Titania and Yantala," J. Non Cryst. Solids 438, 59 (2016), doi:10.1016/j. jnoncrysol.2016.02.009.

- S. Chao, H.-W. Pan, L.-C. Kuo, V. Pierro, M. Principe, I. Pinto, R. DeSalvo, "Nm-Layered Glassy Oxide Composites for 3rd Generation Interferometric Gravitational Wave Detectors," in Optical Interference Coatings 2016, OSA Technical Digest Series (online) (Optical Society of America, 2016), paper MB.10, doi:10.1364/OIC.2016.MB.10.

\section{Bigger optics}

Advanced LIGO's larger (34-cm diameter) optics contribute to increased sensitivity, but posed the additional challenge of maintaining uniform coating thickness over unprecedented large surfaces. Clean rooms and large coating chambers created specifically for building the European interferometer Virgo allowed LMA to meet this challenge.

Pinard and colleagues took "four years to achieve the final performance on the coating thickness uniformity of the end mirrors."

Although ion-beam sputtering is the best technique for producing low-loss coatings, the procedure introduces high internal stress in the coated material. Annealing inside the chamber and afterward, done ex situ in a large oven, helps to reduce absorption and reorganize the amorphous layers.

Australia's Commonwealth Scientific and Industrial Research Organisation (CSIRO) in Sydney, which polished the core optics for Initial LIGO, coated the other optical components for Advanced LIGO. As members of a research group that had focused on multilayer optical coatings since the 1950s, Mark Gross and Anatoli Chtanov developed new methods to apply uniform coatings without the need for a very large chamber.

"Choice of coating materials plays a part, but annealing procedures have a very large effect on minimizing absorption due to lack of perfect stoichiometry, and stress," says Roger Netterfield, former CSIRO senior principal research scientist. Their approach used commercial software to design multilayer stacks that produced acceptable tolerance to thickness errors.

CSIRO also developed an in situ multiwavelength ellipsometric monitoring technique, which analyzes changes in polarization as light reflects from a sample, to measure both coating thickness and refractive index in real time.

\section{Looking ahead}

From shaping and polishing high-precision mirrors, to techniques for applying uniform coatings over large surfaces, LIGO research interfaces directly with the optics community. The story of the 
coatings is still in its early stages. Future chapters will focus on the challenge of reducing thermal noise, without degrading optical absorption.

In the years since Advanced LIGO was constructed, new studies have provided better understanding of how tantala bond angles change when doped with titania. According to Harry, it is only recently that "we're starting to understand what's going on and how we get mechanical loss."

Scientists are investigating new materials as well as titanium doping, using different percentages to maintain optical performance while improving thermal noise. If they can achieve a higher refractive index, they could reduce layer thickness. "If we can reduce total thickness, we can reduce loss," Pinard says.

Silica and tantala offer sufficiently low mechanical loss to meet LIGO's current sensitivity requirements. But future designs might involve more than two materials, perhaps using a low absorption stack on top of the mirror and a low mechanical loss substance, such as amorphous silicon, near the bottom. Nanolayered amorphous glassy oxide composites could exhibit better mechanical and optical properties, and use cryo-friendly materials like hafnia and titania.

And while amorphous oxides are conducive to achieving uniform coating over a large surface, “you wouldn't want to go with amorphous [oxides] if you designed a new, low-loss coating from scratch," Harry notes. If a technique could be developed for growing a uniform crystalline coating on unprecedented large surfaces, aluminum gallium arsenide (AlGaAs) is one potential candidate. Macroscopic quantum mechanics experiments that use optical cavities, albeit with sub-mm diameter mirrors, have achieved good results using AlGaAs coatings. The material is now sold for low thermal noise coatings for precision timing experiments.

Another possibility builds on optical filters with dielectric coatings whose refractive index is varied continuously through graded materials, rather than in steps through interfacial layers. "This opens many possibilities in materials science as a means of reducing both scattering and mechanical noise, yet is based on ideas pursued in the past for coatings using techniques other than ion-beam sputtering," Netterfield says.
Future gravitational-wave observatories could use different designs such as silicon test masses or cryogenically cooled optics. Larger interferometers would also increase signal-to-noise ratio for gravity waves; and future detectors could use different lasers, making way for materials with demonstrated optical properties at other wavelengths.

LIGO's next observation run is scheduled for this Fall, and will commence using the same mirrors and coatings as when LIGO made headlines earlier this year. This time, the two US-based interferometers will be joined by the Virgo interferometer in Pisa, Italy, for the first common datacollecting period with all three instruments operating at improved sensitivity levels.

In the longer term, discussions about thermal noise center around mirrors with lower-loss coatings. Because interferometers detect the amplitude of a wave, a factor of two reduction in thermal noise would translate to a factor of eight in detection rate.

Better understanding of coatings that will not jeopardize optical properties has great potential to improve LIGO sensitivity. "It helps to get the word out about the problem to the materials community," Raab says.

\section{Nano Focus}

Researchers take diamond defect to new depths in magnetic imaging

Titrogen-vacancy (NV) centers are 1 a rising star in the field of highresolution magnetic imaging. Stable enough to capture condensed-matter phenomena at room temperature, these versatile, atom-sized defects are now soaring to new heights in the magnetism community - and descending to new depths. Research groups led by Ania Jayich from the University of California, Santa Barbara, and Patrick Maletinsky from the University of Basel in Switzerland, have independently developed NV-based sensors that can resolve nano-sized magnetic features at temperatures as low as a few degrees above zero Kelvin. With these sensors, condensed-matter physicists now have access to a wider range of magnetic imaging temperatures.

"There's been a big push toward lowtemperature operations because there is a lot of very exciting electronic systems that exist at low temperatures," says Maletinsky, assistant professor of experimental physics. "Graphene, quantum Hall effects, spin Hall effects-there's a big variety." For Maletinsky, who has been pushing the limits of NV-based imaging since his days as a postdoctoral researcher at Harvard University, his group's most recent advance, published in Nature Nanotechnology (doi:10.1038/ NNANO.2016.63), represents an important technological breakthrough.

NV centers are naturally occurring defects in diamond that consist of a substitutional nitrogen atom and a neighboring lattice vacancy. In the laboratory, NV centers are created by ion implanting nitrogen atoms in a piece of diamond,

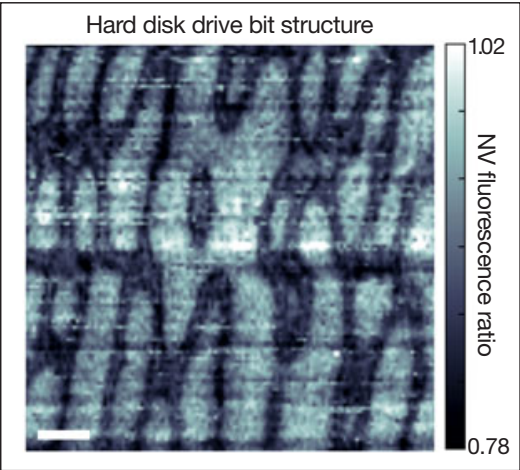

Nitrogen-vacancy (NV) magnetometry image of the bits of a hard disk at $T=6 \mathrm{~K}$. Dark features correspond to the $5.3 \mathrm{G}$ magnetic field contours (2892 MHz RF field). Scale bar, $100 \mathrm{~nm}$. Credit: Nature Nanotechnology.

knocking carbon atoms out of place in the lattice. Each implanted nitrogen atom and associated vacancy together behave as a single quantum spin. This allows scientists to track the energetic signature 